\title{
The Examination of non-pharmacologic treatment of pain after spinal cord injury
}

\author{
Piatt, J. A. ${ }^{1}$, Eldridge, L. ${ }^{1}$, \& Baker, J.M. ${ }^{2}$ \\ ${ }^{1}$ Indiana University Bloomington, School of Public Health, Department of Parks, Recreation and \\ Tourism Studies, ${ }^{2}$ Indiana University School of Medicine
}

Background: One of the primary debilitating secondary health conditions experienced by individuals living with a spinal cord injury $(\mathrm{SCl})$ is chronic pain. Approximately, over $30 \%$ of $\mathrm{SCl}$ patients endure chronic pain after sustaining the injury, and engage in opioid pharmacotherapy as the first form of treatment. The increase in use and misuse of prescribed opioids for chronic pain can lead to both physical and psychological health risks. This danger is exacerbated by the notion that a large percentage of the $\mathrm{SCl}$ population have a pre-existing condition of drug and alcohol abuse and addiction. This study will examine how pain is actually being addressed through non-pharmaceutical methods among the SCl adult population.

Experimental Design: Employing a cross-sectional web survey design with a convenience sample of adults with a SCl. A Qualtrics survey is currently being administered to known individuals with $\mathrm{SCls}$ via email. The survey questions will illuminate what pain management strategies are being implemented in the SCI population within the US.

Anticipated Results: The results from the data will provide insight on what nonpharmacological interventions can be employed in place of or in combination with the pharmacological management. This will allow community-based rehabilitation therapies to incorporate appropriate pain management strategies.

Potential Impact: Non-pharmacological interventions may hold the answer in treating chronic pain in the SCl population by limiting or eliminating the use of opioids. This study will help develop the most appropriate non-pharmacological intervention to test in quasi-experimental clinical trials, and ultimately minimize the use of opioids for chronic pain. 